\title{
DNA damage signaling in response to 5-fluorouracil in three colorectal cancer cell lines with different mismatch repair and TP53 status
}

\author{
BIRGITTE L. ADAMSEN, KATHERINE L. KRAVIK and PAULA M. DE ANGELIS
}

Department of Pathology, Clinic for Diagnostics and Intervention, Oslo University Hospital, Rikshospitalet, Oslo, Norway

Received February 25, 2011; Accepted April 19, 2011

DOI: $10.3892 /$ ijo.2011.1080

\begin{abstract}
We studied patterns of DNA damage signaling and cell cycle response to clinically-relevant (bolus) and high doses of 5-fluorouracil (5-FU) in three colorectal cancer cell lines with differing MMR and TP53 status in an attempt to better understand how 5-FU exerts its cytotoxicity. The ATM/CHEK2/ CHEK1 signaling pathway was not activated in response to bolus 5-FU in the MMR-deficient cell lines HCT116 (TP53proficient or TP53-depleted) and HCT15 (TP53-deficient), consistent with negligible/reparable DNA damage and no cell death. The pattern of DNA damage checkpoint activation in bolus 5-FU-treated HT29 (TP53-deficient/MMR-proficient) cultures suggested SSB formation (CHEK1 activation) followed by DSB formation (CHEK2 activation and increased phosphoH2AX levels), but no cell death suggested that DNA repair capacity was not overwhelmed. High-dose 5-FU treatment led to activation of ATM/CHEK2/TP53 (not CHEK1) in TP53proficient and TP53-depleted HCT116 (later CHEK2 activation relative to TP53-proficient) cultures; HCT15 cultures had ATM activation only. These data and increased phospho-H2AX levels indicated DSB formation; apoptosis was induced in both cell lines indicating irreparable DNA damage. TP53-depleted HCT116 cultures also had DSBs after high-dose 5-FU treatment but experienced a (transient) $\mathrm{G}_{1} / \mathrm{S}$ cell cycle arrest that protected them from apoptosis. TP53 phosphorylation at Ser20/33/37
\end{abstract}

Correspondence to: Dr Paula M. De Angelis, Department of Pathology, Clinic for Diagnostics and Intervention, Oslo University Hospital, Rikshospitalet, Oslo, Norway

E-mail: paula.deangelis@rr-research.no

Abbreviations: RNAi, RNA interference; siRNA, small interfering RNA; 5-FU, 5-fluorouracil; SSB, single-strand break; DSB, doublestrand break; ATM, Ataxia telangiectasia mutated; CHEK1, checkpoint kinase 1; CHEK2, checkpoint kinase 2; H2AX, H2A histone family, member X; CDKN1A, cyclin-dependent kinase inhibitor 1A (p21); BER, base excision repair; MMR, mismatch repair; NER, nucleotide excision repair

Key words: colon cancer, 5-fluorouracil, TP53, RNA interference, DNA damage response, cell cycle alterations was seen in TP53-proficient HCT116 cultures regardless of 5 -FU concentration at $\geq 4 \mathrm{~h}$ following treatment, indicating TP53 stabilization/transcriptional activation. Overall, activation of ATM, CHEK1 and/or CHEK2 and phospho-H2AX levels reflected the nature of 5-FU-induced DNA damage and indicated when DNA damage was significant (5-FU-dose-dependent). DNA repair and cell cycle responses to 5-FU-induced DNA damage were distinctly affected by MMR and TP53 (role in BER/NER) functionalities, but MMR deficiency especially seemed to confer less overall sensitivity to 5-FU.

\section{Introduction}

The nucleoside analogue 5-fluorouracil (5-FU) remains the gold standard as first-line treatment for colorectal, breast and other cancer types (1). The mechanisms of 5-FU cellular toxicity remain poorly understood despite a relatively good understanding of the enzymatic conversion and metabolic fates of 5-FU in vivo (2). The mechanism of 5-FU action involves the inhibition of thymidylate synthase (TS) by fluorodeoxyuridine monophosphate (FdUMP), a metabolic derivative of 5-FU. TS inhibition results in the depletion of intracellular pools of deoxythmidine mono- and triphosphate (dTMP and dTTP) and increases in the relative levels of the normal precursor dUMP and its anabolic derivative, dUTP (1). These nucleotide pool perturbations may result in replication fork stalling and increased misincorporation of dUTP rather than dTTP. FdUMP is also converted into fluorodeoxyuridine triphosphate (FdUTP) which itself is a substrate for DNA polymerases and readily misincorporated into DNA (2).

Increased understanding of the precise nature of the DNA damage induced by $5-\mathrm{FU}$ and the cellular responses to this damage may contribute to a better understanding of 5-FU cytotoxicity. This would be useful for optimization of its clinical effect, since only a small percentage of tumors respond to 5-FU (1). 5-FU-induced DNA damage may arise via the collapse of stalled replication forks leading to single-strand breaks (SSBs) and/or double-strand breaks (DSBs) or other abnormal structures. Excision of misincorporated (potentially mutagenic and miscoding) FdUTP or dUTP from DNA by base excision repair (BER) and possibly mismatch repair (MMR) may also create DSBs if multiple, closely spaced lesions are processed simultaneously $(3,4)$. Additional misincorporation of 
FdUTP or dUTP under conditions of dTTP pool depletion will result in multiple and futile repair cycles and can contribute to a compromise of DNA repair.

DNA damage activates DNA damage checkpoint pathways $(5,6)$, one mediated by ATM through CHEK2 in response to DSBs $(7,8)$ and the other mediated by ATR through CHEK1 in response to bulky DNA lesions and replication fork collapse during S phase (9) as well as SSBs $(6,10)$. The MRN complex (comprised of MRE11, RAD50 and NBS1 subunits) recognizes DSBs and this transient interaction is sufficient enough to recruit ATM (5). ATM, ATR or DNA-PK can activate the effector protein TP53 which plays a major role in DNA damage response $(6,11,12)$. Phosphorylation of serine residues 15,20 , 37 and Thr18 stabilizes TP53 by disrupting the interaction between TP53 and MDM2 $(13,14)$. ATM mainly phosphorylates the Ser15 residue in response to ionizing radiation or chemotherapeutic agents $(15,16)$ whereas ATR phosphorylates both Ser15 and Ser37 residues when cells are treated with UV and inhibitors of synthesis $(17,18)$.

We investigated patterns of DNA damage signaling and cell cycle effects in response to clinically-relevant bolus and high-dose 5-FU treatments in three human colorectal cancer cell lines with differing mismatch repair (MMR) and TP53 status. MMR deficiency can compromise the cellular response to 5-FU and other chemotherapeutic agents (19) as shown in a previous study (19), whereas restoration of the MMR gene $h M L H 1$ in the MMR-deficient HCT116 colon cancer cell line restored 5-FU sensitivity (20). DNA damage checkpoint activation and cell cycle progression patterns were specifically studied in MMR-deficient/TP53-proficient, MMR-deficient/ TP53-deficient and MMR-proficient/TP53-deficient cell lines (HCT116, HCT15 and HT29, respectively). Our data provide new insights into the nature of DNA damage induced by $5-\mathrm{FU}$ and may contribute to a better understanding of 5-FU cytotoxicity.

\section{Materials and methods}

Cell lines, culture conditions and transfection with siRNAs. The following human colorectal cancer cell lines were used: human colon cancer cell line HCT116 [wild-type TP53 gene; MMR-deficient due to lack of the $h M L H 1$ (20-22) and $h M S H 3$ (23) genes, American Type Culture Collection No. CCL-247]; the colorectal cancer cell line HT29 [mutated TP53 gene at codon 273 (24); MMR-proficient (25), American Type Culture Collection No. HTB-38]; and the human colon cancer cell line HCT15 [mutated TP53 gene; mutated CHEK2 gene; MMR deficient due to lack of $h M S H 6$ gene (21,26-28), American Type Culture Collection No. CCL-225]. All cell lines were cultured in RPMI media supplemented with $10 \%$ heat-inactivated fetal bovine serum, $2 \mathrm{mM} \mathrm{L}$-glutamine and $0.05 \mathrm{mg} / \mathrm{ml}$ Gentamicine in a humidified incubator at $37^{\circ} \mathrm{C}$ with $95 \% \mathrm{O}_{2}$ and $5 \% \mathrm{CO}_{2}$. Transfections of the HCT116 cell line with specific siRNAs against TP53 (Dharmacon Inc., Lafayette, CO) and control siRNAs were performed as described previously (29). The human colon cancer cell line HCT116 was transfected with $30 \mathrm{nM}$ siTP53, a siRNA specific for TP53 (Dharmacon Inc.) using Lipofectamine 2000 (Invitrogen, Karlsruhe, Germany). Control cultures were transfected with a non-specific siRNA, $30 \mathrm{nM}$ siControl (Dharmacon Inc.). After transfection at $37^{\circ} \mathrm{C}$ for $1 \mathrm{~h}$, the media were aspirated off and RPMI media supplemented with $2 \mathrm{mM}$ L-glutamine, $0.05 \mathrm{mg} / \mathrm{ml}$ Gentamicine and $20 \%$ heat-inactivated fetal bovine serum was added. To assess transfection efficiencies, cells were co-transfected with a fluorescein isothiocyanate (FITC)-labeled Block-it oligo (Invitrogen, Karlsruhe, Germany); as in our previous study (29), transfection efficiencies were circa $50 \%$.

Drug treatment and viability assays. 5-FU (Calbiochem, Merck Chemicals, Nottingham, UK) was prepared as a $10 \mathrm{mM}$ stock solution in distilled $\mathrm{H}_{2} \mathrm{O}$. The stock solution was diluted in RPMI media to $500 \mu \mathrm{M}$ before use in the experiments. A single 5-FU bolus dose $(500 \mu \mathrm{M})$ was used to treat HCT116, HCT15 and HT29 cultures $(30,31)$. Stepwise dilutions of medium after 5-FU addition were timed to approximate clinical clearance kinetics over a 24 -h period, i.e., the initial 5-FU concentration of $500 \mu \mathrm{M}$ was diluted to $250,100,20,2$ and finally to $0.5 \mu \mathrm{M}$ (effective dose). This in vitro protocol provided a drug concentration profile similar to that obtained in patients after a $600-\mathrm{mg} / \mathrm{m}^{2}$ bolus dose of 5-FU (31). HCT116 (TP53-proficient and TP53-depleted) and HCT15 cultures were also treated with a continuous high-dose of 5-FU $(380 \mu \mathrm{M})$ for purposes of comparison. This concentration has previously been shown to result in alterations in cell cycle progression and in considerable apoptosis induction (29) compared to bolus 5-FU treatment. Control cultures received no treatment of any kind. Cell cultures were harvested by trypsinization at $0,0.5,1,2,4,6,8,12,24$ and $48 \mathrm{~h}$ (HT29 and HCT15 cultures) after drug addition for further analyses. Viability and total cell numbers were assessed using standard trypan blue viability assays. Data presented are the results of at least two replicate experiments.

Cell cycle analyses. Harvested cells were fixed in $80 \%$ methanol and stored at $-20^{\circ} \mathrm{C}$ until analysis. Methanol-fixed cell suspensions of isolated nuclei were stained with propidium iodide (PI) using Vindelov's procedure (32). Cell cycle analyses and doublet discriminations were performed using a FACSCalibur laser flow cytometer (BD Biosciences, Palo Alto, CA) as previously described (29). Percentages of cells in the $\mathrm{G}_{1}, \mathrm{~S}$ and $\mathrm{G}_{2} / \mathrm{M}$ phases of the cell cycle were estimated using WinCycle software (Phoenix Flow Systems, San Diego, CA).

Western blot analyses. Harvested monolayer cells were pooled with floating cells and boiled for 5-10 min in standard Laemmli buffer. Protein concentrations were determined using the Bradford assay (Bio-Rad Laboratories, Hercules, CA). Protein (10 or $15 \mu \mathrm{g}$ ) per lane (5 $\mu \mathrm{g}$ of protein molecular weight standards) were loaded and run on 5, 7.5 or 12\% SDS-polyacrylamide gels. Separated proteins were transferred to PVDF membranes (Criterion, Bio-Rad Laboratories). Non-specific binding was blocked using $5 \%$ non-fat milk for $1 \mathrm{~h}$ at room temperature, and then the membranes were incubated with the primary mouse or rabbit antibodies overnight at $4^{\circ} \mathrm{C}$. The following primary antibodies were used: mouse monoclonals TP53 (Ab-2) and CDKN1A (Calbiochem, Cambridge, MA); rabbit polyclonals phospho-TP53 (Ser15), phospho-TP53 (Ser33), phospho-TP53 (Ser37), cleaved PARP, CHEK1, phospho-CHEK1 (Ser317), phospho-CHEK1 (Ser345), CHEK2, phospho-CHEK2 (Thr68), phospho-CHEK2 (Thr387), phospho-ATM (Ser1981) 
A Bolus 5-FU-treated siTP53- and siControl-transfected HCT116 cultures
B Bolus 5-FU-treated siTP53- and siControl-transfected HCT116 cultures
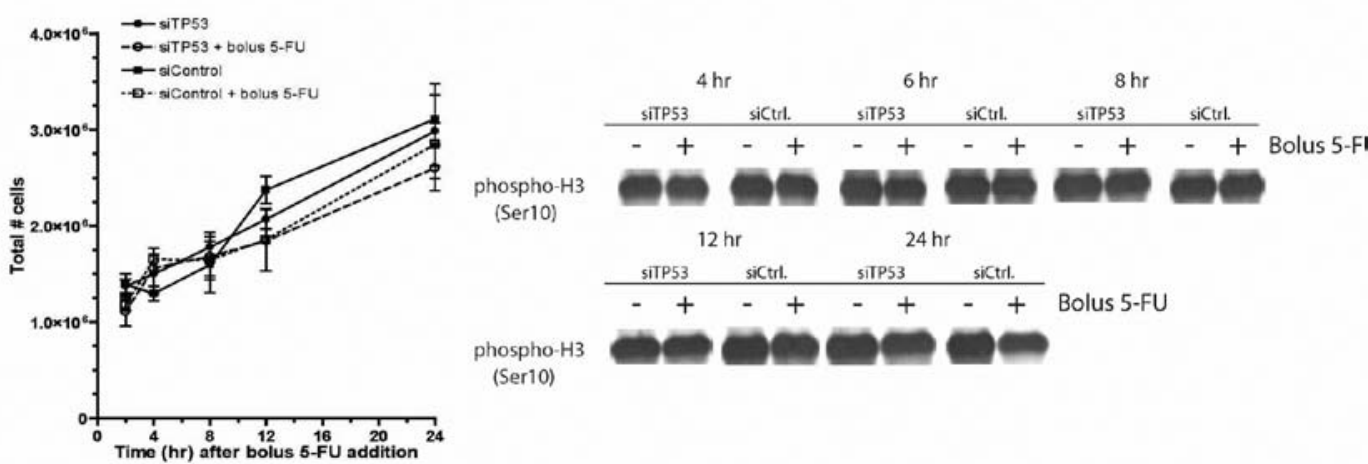

c
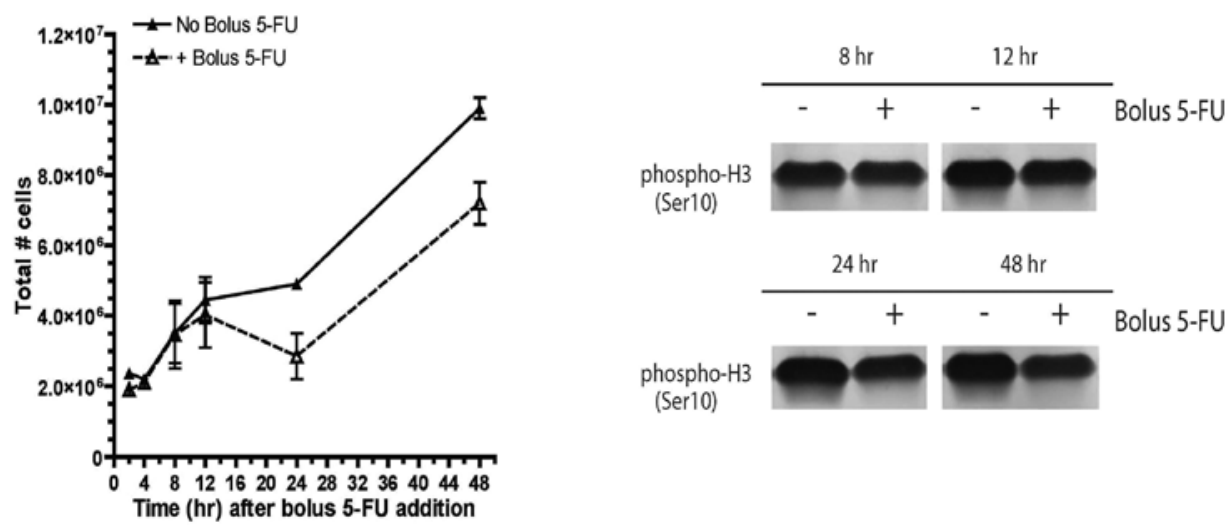

(Ser10)
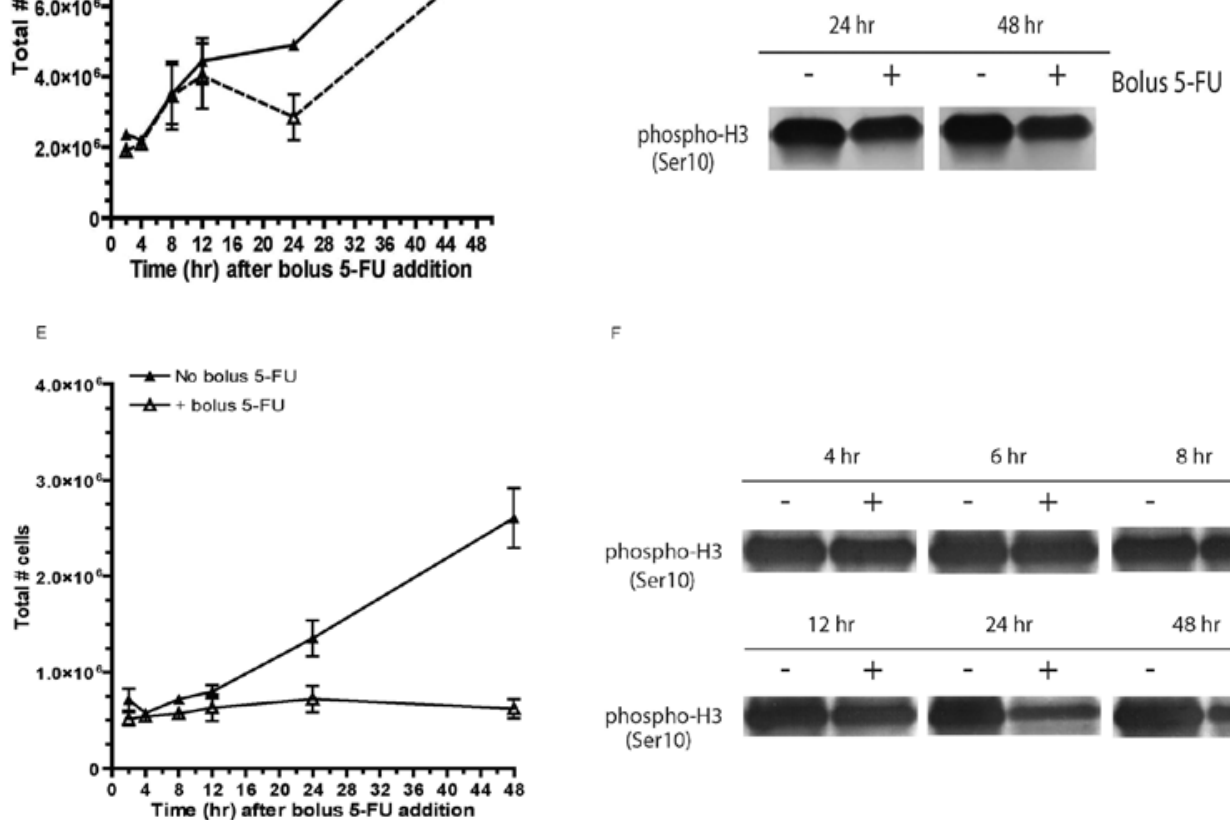

F

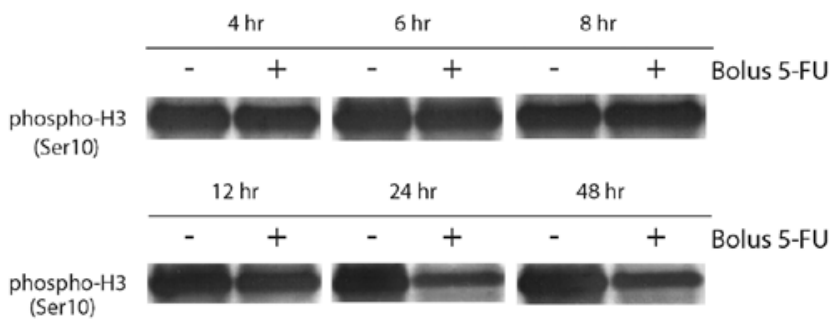

Figure 1. Total cell counts and mitotic activity in bolus 5-FU-treated HCT116, HCT15 and HT29 cultures. Total cell counts for HCT116 (A), HCT15 (C) and HT29 (E) cultures were measured using standard trypan blue viability assays. Data (means \pm SEM) are from two replicate experiments. The Western blots for phospho-H3 (Ser10) (biomarker of mitotic activity) are shown for bolus 5-FU-treated TP53-depleted (siTP53-transfected) and TP53-proficient (siControltransfected) HCT116 cultures (B), bolus 5-FU-treated HCT15 cultures (D) and bolus 5-FU-treated HT29 cultures (F).

(10H11.E12), MRE11 rabbit monoclonal (31H4), NBS1 (p95), RAD50 (Cell Signaling Technology, Danvers, MA); mouse monoclonals H2AX (Ser139) and ATM, rabbit polyclonal phospho-H3 (Ser10) (Upstate, Lake Placid, USA); rabbit polyclonal phospho-TP53 (Ser20) (R\&D Systems, MN, USA); mouse monoclonal UDG (k1C12) (Santa Cruz Biotechnology, Santa Cruz, CA). All antibodies were used at working concentrations of $1-2 \mu \mathrm{g} / \mathrm{ml}$. Blots were incubated with biotinylated goat anti-rabbit IgG or biotinylated horse anti-mouse IgG for $1 \mathrm{~h}$ followed by incubation with a streptavidin-biotinylatedalkaline phosphatase complex for $1 \mathrm{~h}$ and colorimetric development. Protein levels were quantified using UnScanit gel software version 5.1 for Windows (Silk Scientific Inc., Orem, UT). Equal protein loading was confirmed by a mouse monoclonal actin (C-2) antibody (Santa Cruz Biotechnology).

Statistical analyses. Statistical analyses and plots were generated using GraphPad Prism 4.0 software (GraphPad Software, Inc., San Diego, CA).

\section{Results}

We assessed cell growth, patterns of DNA damage response and cell cycle progression in response to bolus and continuous high- 
A High-dose 5-FU-treated TP53-depleted and and TP53-proficient HCT116 cultures

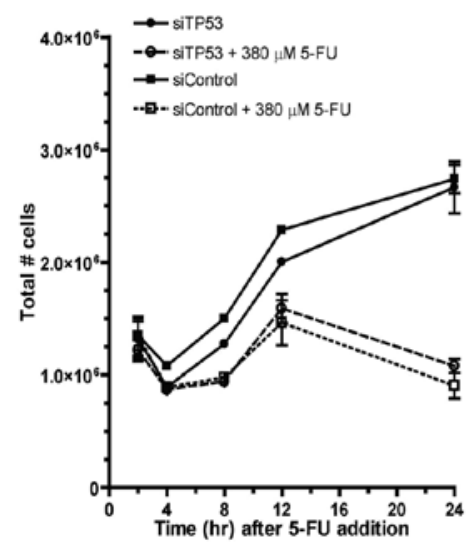

C High-dose 5-FU-treated HCT15 cultures

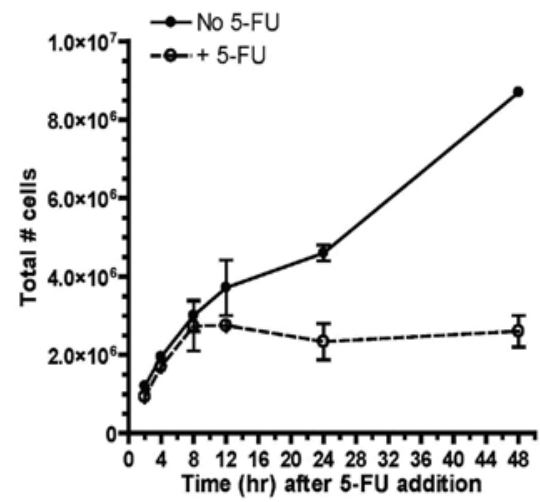

B High-dose 5-FU-treated TP53-depleted and and TP53-proficient HCT116 cultures

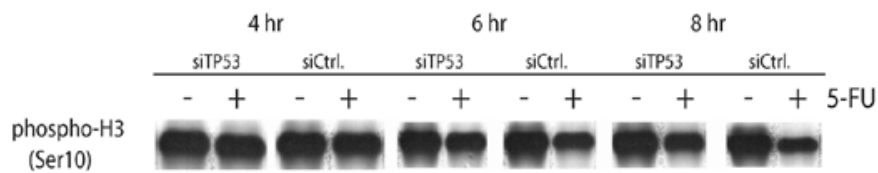

(Ser10)

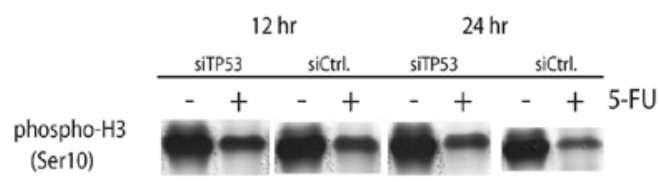

D High-dose 5-FU-treated HCT15 cultures

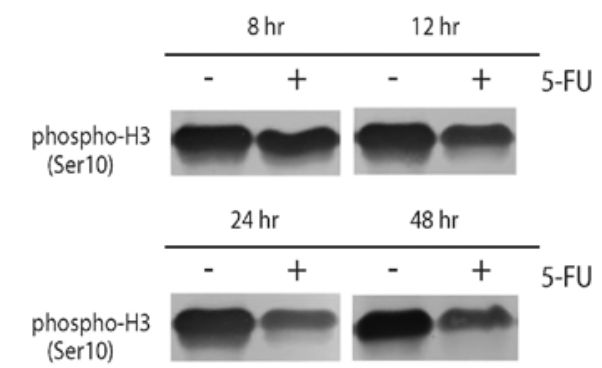

Figure 2. Total cell counts and mitotic activity in high-dose 5-FU-treated HCT116 and HCT15 cultures. Total cell counts for HCT116 (A) and HCT15 (C) cultures were measured using standard trypan blue viability assays. Data (means \pm SEM) are from two replicate experiments. The Western blots for phospho-H3 (Ser10) are shown for high-dose 5-FU-treated TP53-depleted- and TP53-proficient HCT116 cultures (B) and high-dose 5-FU-treated HCT15 cultures (D).

dose 5-FU in different TP53 and MMR proficiency settings corresponding to the following cell lines: i) MMR-deficient, TP53-proficient (HCT116 cell line); ii) MMR-deficient, TP53-deficient (HCT15 cell line and HCT116 cell line transiently depleted for TP53 via RNA interference); and iii) MMR-proficient, TP53-deficient (HT29 cell line).

Cell growth after 5-FU treatment (bolus and high-dose). TP53-proficient and TP53-depleted HCT116 cultures treated with bolus 5-FU had similar total cell counts and levels of phospho-H3 (Ser10) (a marker of mitotic activity) prior to $12 \mathrm{~h}$ compared to their respective untreated controls (Fig. 1A, B). By 24 h however, only the drug-treated TP53-proficient cultures had (moderately) decreased mitotic activity as indicated by reduced phospho-H3 (Ser10) levels (Fig. 1B). Growth inhibition was evident after $12 \mathrm{~h}$ in bolus 5-FU-treated HCT15 cultures (Fig. 1C), consistent with decreased phospho-H3 (Ser10) levels (Fig. 1D); they began to proliferate again between 24 and $48 \mathrm{~h}$ but still demonstrated noticeable differences in growth at $48 \mathrm{~h}$ compared to control cultures, consistent with decreased levels of phospho-H3 (Fig. 1C, D). Growth inhibition was evident between 4 and $8 \mathrm{~h}$ in bolus 5-FU treated HT29 cultures (Fig. 1E) and persisted throughout the experimental time period; phospho-H3 (Ser10) levels in these cultures were considerably reduced at 24 and $48 \mathrm{~h}$, indicative of reduced mitotic activity (Fig. 1F).

TP53-proficient and TP53-depleted HCT116 cultures treated with a high-dose of 5-FU showed growth inhibition between 4 and $8 \mathrm{~h}$ that persisted at $24 \mathrm{~h}$ consistent with decreasing phospho-H3 (Ser10) levels over the 24-h period (Fig. 2A, B). TP53-proficient cultures treated with continuous high-dose 5-FU tended to have stronger growth inhibition and lower phospho-H3 (Ser10) levels compared to correspondingly-treated TP53-depleted cultures. In HCT15 cultures exposed to high-dose 5-FU, growth inhibition was evident at $12 \mathrm{~h}$, consistent with decreased phospho-H3 (Ser10) levels; this persisted throughout the experimental time course (Fig. 2C, D).

Patterns of DNA damage response and cell cycle progression after 5-FU treatment (bolus and high-dose)

HCT116 cell line (MMR-deficient/TP53-proficient). ATM (Ser1981), CHEK2 (Thr68/387) and CHEK1 (Ser317/345) were not activated in bolus 5-FU-treated TP53-proficient or 
A Bolus 5-FU-treated siTP53-transfected and siControl-transfected HCT116 cultures

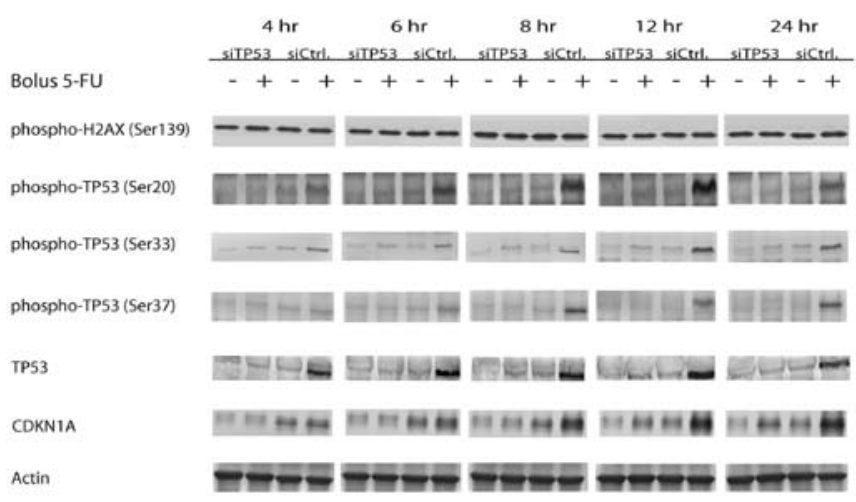

B Bolus 5-FU-treated siTP53- and siControl-transfected $\mathrm{HCT} 116$ cultures

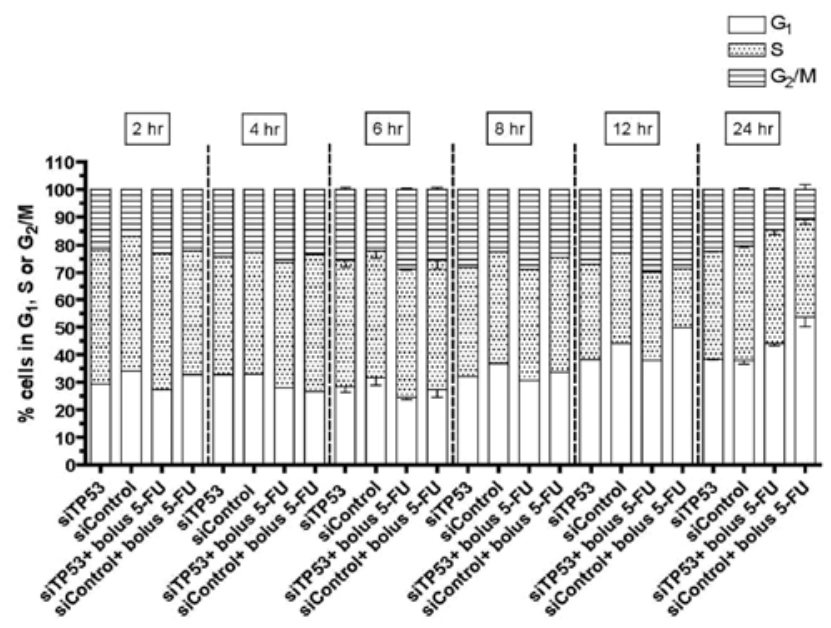

Figure 3. Activation of DNA damage checkpoint proteins and cell cycle progression in bolus 5-FU-treated HCT116 cultures. (A) Western blot showing levels of DNA damage checkpoint activation proteins for untreated and bolus 5-FU-treated TP53-depleted (siTP53-transfected) and TP53-proficient (siControl-transfected) HCT116 cultures for the indicated times. Actin was used as a loading control. (B) Cell cycle progression for the same cultures.

TP53-depleted (via RNA interference) HCT116 cultures at any time-point, and no TP53 activation (at Ser15/20/33/37) was seen prior to $4 \mathrm{~h}$ (data not shown). TP53 activation (Ser20/33/37) and CDKN1A induction became evident in bolus 5-FU-treated TP53-proficient cultures at about $4 \mathrm{~h}$, but not in TP53-depleted cultures at any time-point (Fig. 3A). CHEK1 and CHEK2 were constitutively expressed in bolus 5-FU-treated TP53-proficient and TP53-depleted cultures. Phospho-H2AX (Ser139) levels were not altered in response to bolus 5-FU treatment in TP53-proficient or TP53-depleted HCT116 cultures at any time-point (Fig. 3A). Constitutive levels of MRE11, NBS1, RAD50, UDG and cleaved PARP were not altered at any time-point in bolus 5-FU-treated TP53proficient or TP53-depleted HCT116 cultures (data not shown). There were no significant alterations in cell cycle progression in bolus 5-FU-treated TP53-depleted cultures during the 24-h time period relative to bolus 5-FU-treated TP53-proficient cultures that had slightly larger $\mathrm{G}_{1}$ and smaller $\mathrm{G}_{2}$ phase fractions by $24 \mathrm{~h}$ (Fig. 3B).

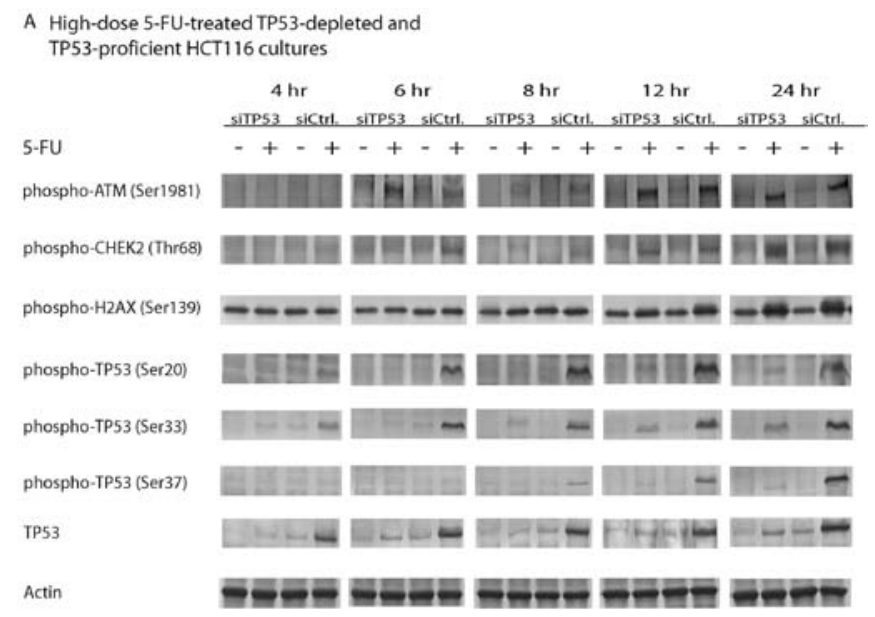

B High-dose 5-FU-treated TP53-depleted and TP53-proficient HCT116 cultures

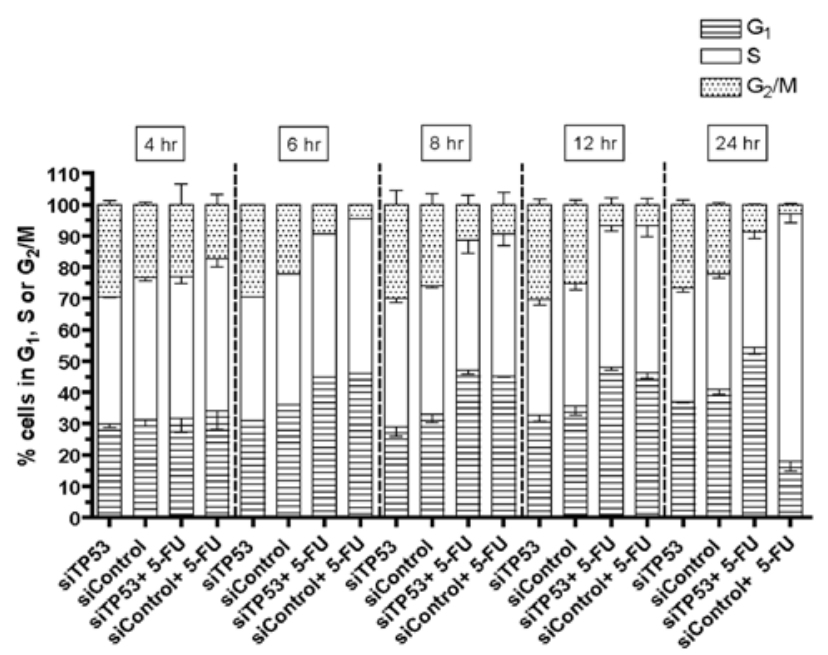

Figure 4. Activation of DNA damage checkpoint proteins and cell cycle progression in high-dose 5-FU-treated HCT116 cultures. (A) Western blot showing levels of DNA damage checkpoint activation proteins for untreated and high-dose 5-FU-treated TP53-depleted and TP53-proficient HCT116 cultures for the indicated times. Actin was used as a loading control. (B) Cell cycle progression for the same cultures.

Strong ATM activation in response to continuous high-dose 5-FU treatment was seen in TP53-depleted HCT116 cultures from $6 \mathrm{~h}$ and in TP53-proficient HCT116 cultures from $12 \mathrm{~h}$ (Fig. 4A). CHEK2 (Thr68) activation was seen in both drugtreated TP53-proficient and TP53-depleted HCT116 cultures at $12 \mathrm{~h}$, but was first seen in TP53-proficient cultures at $6 \mathrm{~h}$ (Fig. 4A). CHEK1 activation was not seen at any time-point; the constitutive levels of CHEK1, CHEK2, NBS1, RAD50, MRE11 and UDG were not altered as a result of high-dose 5-FU in either TP53-proficient or TP53-depleted HCT116 cultures relative to respective untreated controls (data not shown). Phospho-H2AX (Ser139) levels in drug-treated TP53-proficient and TP53-depleted HCT116 cultures were strongly increased at $24 \mathrm{~h}$, but the TP53-proficient cultures had larger increases in the levels of this protein already at $12 \mathrm{~h}$ compared to TP53depleted cultures (Fig. 4A). TP53 was strongly phosphorylated at Ser20/33/37 in TP53-proficient HCT116 cultures from $4 \mathrm{~h}$ (Fig. 4A) and CDKN1A induction was seen between 6 and 
A Bolus 5-FU-treated $\mathrm{HCT} 15$ cultures

Bolus 5-FU

phospho-H2AX (Ser139)

phospho-TP53 (Ser20)

phospho-TP53 (Ser33)

phospho-TP53 (Ser37)

Actin

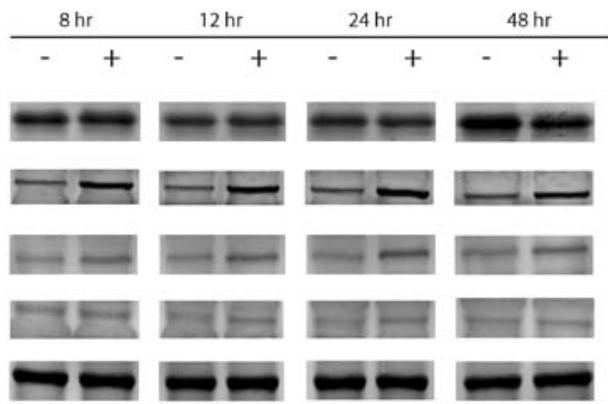

B Bolus 5-FU-treated HCT15 cultures

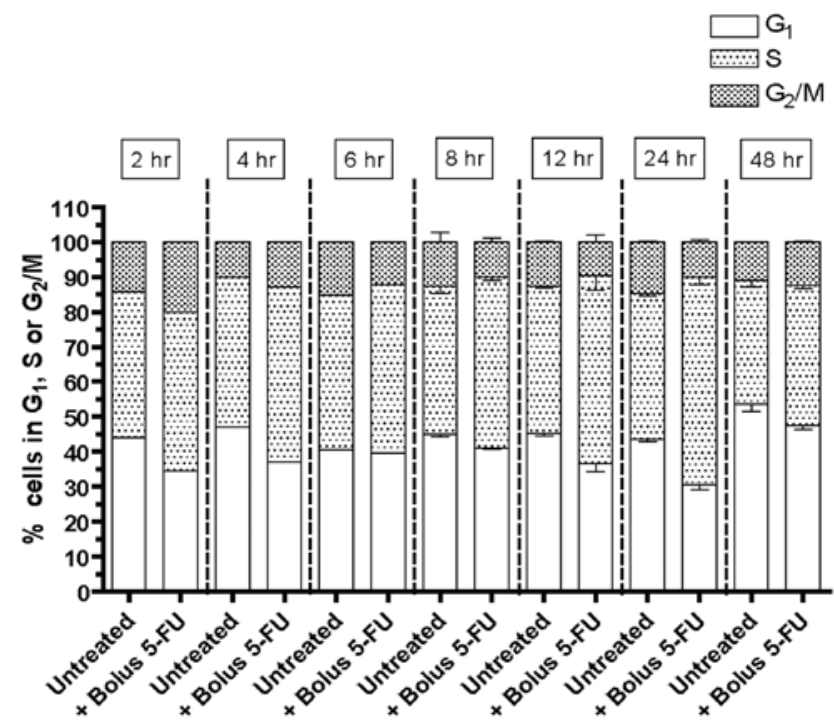

Figure 5. Activation of DNA damage checkpoint proteins and cell cycle progression in bolus 5-FU-treated HCT15 cultures. (A) Western blot showing levels of DNA damage checkpoint activation proteins for untreated and bolus 5-FU-treated HCT15 cultures for the indicated times. Actin was used as a loading control. (B) Cell cycle progression for the same cultures.

$24 \mathrm{~h}$ (data not shown) but not at earlier time-points. TP53 activation and CDKN1A induction in drug-treated TP53-depleted cultures was negligible (the low levels of TP53 and corresponding observed activation in these cultures was due to the presence of untransfected cells that responded to high-dose 5-FU). The highest levels of cleaved PARP were observed in drug-treated TP53-proficient HCT116 cultures at $24 \mathrm{~h}$ relative to high-dose 5-FU-treated TP53-depleted HCT116 cultures and untreated controls (data not shown), consistent with our earlier findings (29). Different patterns of cell cycle progression were seen in TP53-proficient and TP53-depleted HCT116 cultures following 24-h exposure to high-dose 5-FU. TP53-proficient HCT116 cultures had large $S$ phase arrests at $24 \mathrm{~h}$, whereas TP53-depleted HCT116 cultures had larger $\mathrm{G}_{1}$ fractions and smaller S phase fractions at $24 \mathrm{~h}$ (Fig. 4B) consistent with our earlier study (29).

HCT15 cell line (MMR-deficient/TP53-deficient). Activation of ATM, CHEK2 or CHEK1 in response to bolus 5-FU was not

A High-dose 5-FU treated HCT15 cultures

$5-\mathrm{FU}$

phospho-H2AX (Ser139)

phospho-TP53 (Ser20)

phospho-TP53 (Ser33)

phospho-TP53 (Ser37)

Actin

\begin{tabular}{ccccc}
$8 \mathrm{hr}$ & $12 \mathrm{hr}$ & $24 \mathrm{hr}$ & $48 \mathrm{hr}$ \\
\hline- & + & - & + & -
\end{tabular}
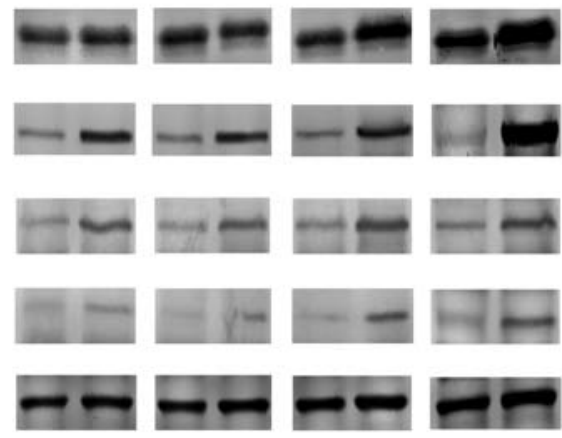

B High-dose 5-FU treated HCT15 cultures

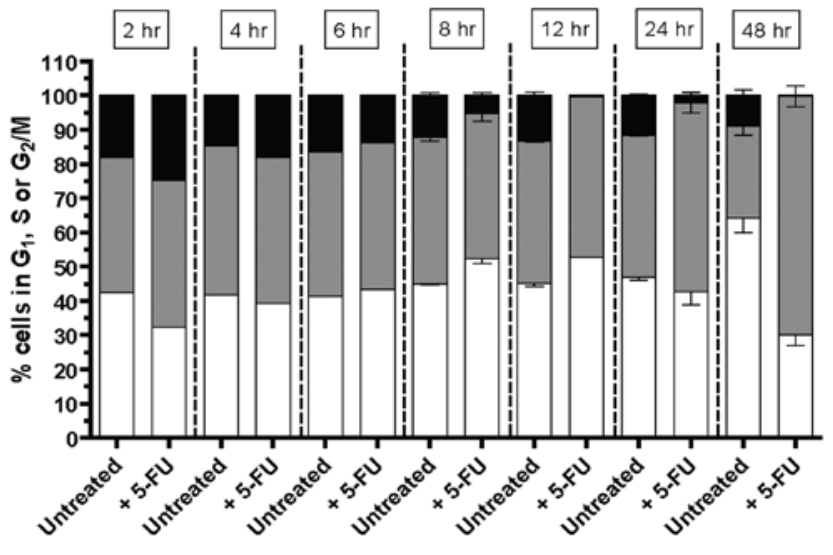

Figure 6. Activation of DNA damage checkpoint proteins and cell cycle progression high-dose 5-FU-treated HCT15 cultures. (A) Western blot showing levels of DNA damage checkpoint activation proteins for untreated and high-dose 5-FU-treated HCT15 cultures for the indicated times. Actin was used as a loading control. (B) Cell cycle progression for the same cultures.

seen (data not shown), and phospho-H2AX (Ser139) levels were unchanged compared to untreated control levels (Fig. 5A). No induction of CDKN1A was detected in HCT15 cultures treated with bolus 5-FU (data not shown), consistent with the HCT15 cell line having non-functional TP53. TP53 was strongly phosphorylated at Ser20 and moderately phosphorylated at Ser33 (but not at Ser37) from $8 \mathrm{~h}$ and onward (Fig. 5A) but not at earlier time-points (data not shown). Larger $\mathrm{S}$ phase fractions and smaller $\mathrm{G}_{1}$ fractions were seen at $24 \mathrm{~h}$ compared to untreated controls (Fig. 5B) consistent with growth inhibition. However, by $48 \mathrm{~h}$, the sizes of S phase fractions in treated cultures were very similar to those seen in untreated control cultures, $G_{1}$ fractions had increased (Fig. 5B), and total cell counts had also increased (Fig. 1C), indicating that proliferation had resumed. Similar cleaved PARP levels in drug-treated and control cultures indicated negligible cell death (data not shown) which also suggests that DNA damage was negligible or easily reparable in these bolus 5-FU-treated cultures. 
A Bolus 5-FU-treated HT29 cultures

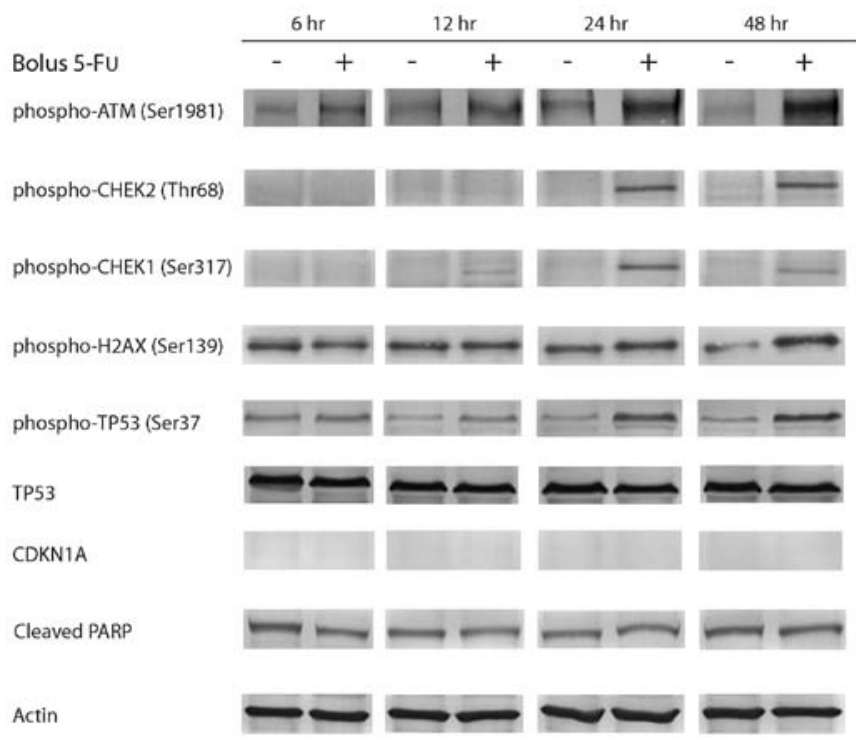

B. Bolus 5-FU-treated HT29 cultures

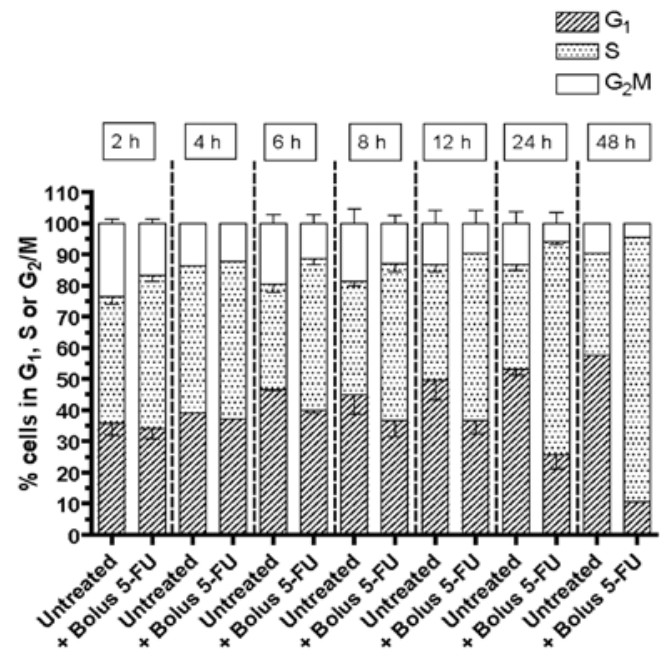

Figure 7. Activation of DNA damage checkpoint proteins and cell cycle progression in bolus 5-FU-treated HT29 cultures. (A) Western blot showing levels of DNA damage checkpoint activation proteins for untreated and bolus 5-FU-treated HT29 cultures for the indicated times. Actin was used as a loading control for the indicated times. (B) Cell cycle progression for the same cultures.

HCT15 cultures treated with a continuous high-dose of 5 -FU showed activation of ATM at $24 \mathrm{~h}$ but not at other time-points and no activation of CHEK2 or CHEK1 (data not shown). The highest levels of phospho-H2AX (Ser139) were observed at $48 \mathrm{~h}$ (Fig. 6A). TP53 phosphorylation was strongest at Ser20 and was detectable at $8 \mathrm{~h}$ (Fig. 6A). CDKN1A was not induced at any time-point consistent with this cell line having non-functional TP53. Cleaved PARP levels were strongly increased at $48 \mathrm{~h}$, indicative of cell death (data not shown). A large population of $\mathrm{S}$ phase-arrested cells was seen at $48 \mathrm{~h}$ (Fig. 6B), consistent with growth inhibition (Fig. 2C, D). The sizes of $\mathrm{G}_{2} / \mathrm{M}$ fractions continued to decrease during the experimental time period, suggesting that cells in $G_{2} / M$ phase underwent cell death.
HT29 cell line (MMR-proficient/TP53-deficient). The HT29 cell line expresses high levels of (mutated and non-functional) TP53 and lacks CDKN1A (Fig. 7A). Prior to $6 \mathrm{~h}$, activation of ATM, CHEK2 or CHEK1 in response to bolus 5-FU was not seen, and phospho-H2AX (Ser139) levels were unchanged relative to untreated control levels (data not shown). ATM activation was first detected at $6 \mathrm{~h}$ and was strongest at $48 \mathrm{~h}$ (Fig. 7A), whereas activation of CHEK2 (Thr68) was first detected at 24 and $48 \mathrm{~h}$ (Fig. 7A). The DNA damage response to bolus 5-FU in these cultures was thus a late response. Weak CHEK1 activation at Ser317 was seen at 12 and $48 \mathrm{~h}$ but was strongest at $24 \mathrm{~h}$. The highest levels of phospho-H2AX (Ser139) in drug-treated HT29 cultures were seen at 48 h (Fig. 7A). CHEK1 and CHEK2 expressions were constitutive at all timepoints (data not shown). TP53 phosphorylation at Ser 37 (but not at Ser15/20/33) was seen at 24 and $48 \mathrm{~h}$. Cleaved PARP levels in untreated and drug-treated cultures were similar throughout the experimental time period (Fig. 7A) indicating lack of apoptosis induction; constitutive levels of MRE11, NBS1, ATM and RAD50 were also unchanged relative to untreated controls (data not shown). Dramatic increases in the sizes of $\mathrm{S}$ phase fractions over the course of the experimental time period were seen in drug-treated cultures relative to untreated controls, reflecting an $\mathrm{S}$ phase cell cycle arrest that persisted at $48 \mathrm{~h}$ (Fig. 7B), consistent with persistent growth inhibition (Fig. 1E, F). Table I summarizes the data for DNA damage signaling and cell cycle responses in colorectal cancer cell lines treated with bolus and/or high-dose 5-FU.

\section{Discussion}

DNA damage signaling and cell cycle responses to clinicallyrelevant and non-clinically-relevant 5-FU treatment were investigated in three different MMR/TP53 settings: MMRdeficient/TP53-proficient (HCT116 cell line); MMR-deficient/ TP53-deficient (HCT15 cell line as well as the HCT116 cell line transiently depleted for TP53); and MMR-proficient/ TP53-deficient (HT29 cell line). Clinically-relevant bolus 5-FU treatment did not lead to SSB or DSB formation in either MMR-deficient cell line (regardless of TP53 proficiency) as indicated by lack of activation of the ATM/CHEK2/ CHEK1 signaling pathway at any experimental time-point. An interesting question is whether MMR-deficient cell lines are completely lacking MMR activity. A recent report has shown that loss of $h M L H 1$ or $h M S H 2$ leads to complete loss of MMR activity (33). The HCT15 cell line is hMSH6 deficient, whereas the HCT116 cell line is deficient both for $h M L H 1$ and $h M S H 3$, suggesting that the HCT116 cell line is completely MMR-deficient compared to HCT15. Thus, MMR as a repair mechanism for excision of misincorporated FdUTP or dUTP from DNA was most likely lacking in the HCT116 cell line and compromised but not completely lacking in the HCT15 cell line. However, both BER and/or NER enzymes could repair this type of DNA damage; a role for TP53 in both BER and NER has previously been reported (34-36). Since the HCT116 cell line is TP53-proficient, it is suggested that BER and/or NER are functional in this cell line and could remove misincorporated FdUTP or dUTP from DNA. BER (and NER) would most likely be compromised in the TP53-deficient HCT15 and HT29 cell lines and the TP53- 
Table I. DNA damage signaling and cell cycle responses in colorectal cancer cell lines treated with bolus and/or high-dose 5-FU.

\begin{tabular}{|c|c|c|c|c|c|c|c|}
\hline \multirow[b]{2}{*}{$\begin{array}{l}\text { Cellular phenotype } \\
\text { (during } 24-48 \mathrm{~h}^{\mathrm{a}} \text { ) }\end{array}$} & \multicolumn{4}{|c|}{ Bolus 5-FU } & \multicolumn{3}{|c|}{ High-dose 5-FU } \\
\hline & HCT116 & $\begin{array}{c}\text { HCT116 } \\
\text { (TP53-depleted) }\end{array}$ & $\mathrm{HCT} 15^{\mathrm{b}}$ & HT29 & HCT116 & $\begin{array}{c}\text { HCT116 } \\
\text { (TP53-depleted) }\end{array}$ & HCT15 \\
\hline Complete growth inhibition & No & No & No & $\begin{array}{c}\text { Yes } \\
\text { (by } 24 \mathrm{~h} \text { ) }\end{array}$ & $\begin{array}{c}\text { Yes } \\
\text { (by } 24 \mathrm{~h} \text { ) }\end{array}$ & $\begin{array}{c}\text { Yes } \\
\text { (by } 24 \mathrm{~h} \text { ) }\end{array}$ & $\begin{array}{c}\text { Yes } \\
\text { (by } 24 \mathrm{~h} \text { ) }\end{array}$ \\
\hline Cell cycle arrest $(\%$ cells \pm SD) & No & No & $\begin{array}{l}\text { S phase } \\
(59 \pm 3)\end{array}$ & $\begin{array}{l}\text { S phase } \\
(85)\end{array}$ & $\begin{array}{l}\text { S phase } \\
(79 \pm 5)\end{array}$ & $\begin{array}{c}\mathrm{G}_{1} / \mathrm{S} \text { transition } \\
(54 \pm 4)\end{array}$ & $\begin{array}{l}\text { S phase } \\
(70 \pm 4)\end{array}$ \\
\hline ATM phosphorylation (Ser1981) & Absent & Absent & Absent & Present & Present & Present & Present \\
\hline CHEK1 phosphorylation (Ser317) & Absent & Absent & Absent & Present & Absent & Absent & Absent \\
\hline CHEK2 phosphorylation (Thr68) & Absent & Absent & Absent & Present & Present & Present & Absent \\
\hline TP53 phosphorylation (Ser15) & Absent & Absent & $\mathrm{ND}^{\mathrm{c}}$ & Absent & Absent & Absent & $\mathrm{ND}$ \\
\hline TP53 phosphorylation (Ser20) & Present & Absent & Present & Absent & Present & Absent & Present \\
\hline TP53 phosphorylation (Ser33) & Present & Absent & Present & Absent & Present & Absent & Present \\
\hline TP53 phosphorylation (Ser37) & Present & Absent & Absent & Present & Present & Absent & Present \\
\hline TP53 & Present & Absent & ND & Absent & Present & Absent & ND \\
\hline CDKN1A & Present & Absent & Absent & Absent & Present & Absent & Absent \\
\hline H2AX phosphorylation (Ser139) & No change & No change & No change & $\begin{array}{l}\text { Increased at } \\
24 \text { and } 48 \mathrm{~h}\end{array}$ & $\begin{array}{l}\text { Increased at } \\
12 \text { and } 24 \mathrm{~h}\end{array}$ & $\begin{array}{l}\text { Increased at } \\
12 \text { and } 24 \mathrm{~h}\end{array}$ & $\begin{array}{l}\text { Increased at } \\
24 \text { and } 48 \mathrm{~h}\end{array}$ \\
\hline $\begin{array}{l}\text { Apoptosis (increase in cleaved } \\
\text { PARP levels) }\end{array}$ & No change & No change & No change & No change & $\begin{array}{l}\text { 3.7-fold } \\
\text { increase }^{\mathrm{d}}\end{array}$ & No change & $\begin{array}{l}\text { 1.7-fold } \\
\text { increase }^{\mathrm{e}}\end{array}$ \\
\hline
\end{tabular}

${ }^{a}$ HCT 116 cultures, for $24 \mathrm{~h}$; HT29 and HCT15 cultures, for 48 h; all phenotypes relative to untreated controls at same time-points. ${ }^{\text {} H C T 116, ~ T P 53-p r o f i c i e n t ~ a n d ~}$ MMR-deficient; HCT15, TP53-deficient and MMR-deficient; HT29, TP53-deficient and MMR-proficient. ${ }^{\mathrm{N} D}$, not determined. ${ }^{\mathrm{d}}$ Relative increases in cleaved PARP levels in drug-treated TP53-proficient HCT116 cultures at $24 \mathrm{~h}$ compared to untreated controls. ${ }^{\mathrm{e}}$ Relative increases in cleaved PARP levels in drug-treated HCT15 cultures at $48 \mathrm{~h}$ compared to untreated controls.

depleted HCT116 cell line. TP53 was phosphorylated at Ser 20/33/37 at $\geq 4 \mathrm{~h}$ in bolus-treated TP53-proficient HCT116 cultures, consistent with stabilization of TP53/inhibition of the TP53-MDM2 complex leading to its transcriptional activation (37). It is suggested that BER (or NER) is sufficient to deal with the bolus-5-FU-induced DNA damage (FdUTP/dUTP misincorporation) in the absence of functional MMR in the TP53-proficient HCT116 cell line, whereas some amount of MMR activity is sufficient to deal with bolus-5-FU-induced DNA damage in the TP53-deficient HCT15 cell line in which BER and NER activity are likely compromised. If there is no MMR activity at all in the HCT15 cell line then it is not clear how it dealt with bolus 5-FU-induced DNA damage in the present study considering that it is also TP53-deficient. Our DNA damage response data are consistent with negligible apoptosis in bolus 5-FU-treated HCT116 and HCT15 cultures regardless of MMR or TP53 status, suggesting that DNA repair capacity was not overwhelmed by high levels of irreparable damage. An interesting question is how TP53 is activated in the absence of activated CHEK1 or CHEK2. Potential activators include BER-associated APEX1 (also known as REF-1) (38,39), p38 kinase (40), ATR or DNA-PK $(18,41,42)$, but this remains unclear since we have not investigated expression of these proteins. Clinically-relevant bolus 5-FU treatment led to SSB and DSB formation in the MMR-proficient/TP53-deficient HT29 cultures as indicated by activation of ATM, CHEK1 and subsequently CHEK2. This was a late response ( $\geq 4 \mathrm{~h}$ after treatment), suggesting that MMR activity was initially able to deal with FdUTP and dUTP misincorporation in a BER/NER-compromised setting, but that over time MMR was overwhelmed which led to stalled replication fork collapse and SSBs (that could not be repaired due to compromised BER) and subsequently DSBs. This is supported by our data showing that in response to bolus 5-FU treatment CHEK1 activation was observed from $12 \mathrm{~h}$ onward (SSB formation), concomitant with (presumed) CHEK1-mediated $\mathrm{S}$ phase arrests protective against apoptosis (43), whereas CHEK2 activation, similarly to elevated phospho-H2AX, appeared at $24 \mathrm{~h}$. Phosphorylation of H2AX is a marker of DSBs (damage-induced DSBs or DSBs caused by apoptosis) $(44,45)$. As there was little to no apoptosis in these cultures at this time-point, the elevated phospho- $\mathrm{H} 2 \mathrm{AX}$ levels reflect 5-FU-induced DSBs and not DSBs resulting from apoptosis. Negligible apoptosis also indicates that DNA repair activity was not overwhelmed. Our findings are novel as they demonstrate that treatment of HT29 cultures with a clinically-relevant bolus 5-FU dose results in the activation of different DNA damage checkpoint proteins reflecting the (sequential) formation of stalled replication forks, SSBs and DSBs. Our findings also indicate that MMR activity alone was not sufficient (BER activity was probably necessary) to deal with SSB damage (resulting from collapse of stalled replication forks) in the HT29 cell line.

High-dose 5-FU treatment led to the formation of DSBs in both the TP53-proficient and TP53-depleted HCT116 cultures as indicated by activation of the ATM-CHEK2-TP53 
signaling pathway (strongest activation seen at 12 and $24 \mathrm{~h}$ ) CHEK1 activation was not seen at any time-point. CHEK2 activation occurred later in TP53-depleted HCT116 cultures than in TP53-proficient cultures, suggesting that DNA damage sensing was delayed in the TP53-depleted cultures. TP53 activation at Ser20/33/37 was seen in drug-treated TP53-proficient HCT116 cultures, suggesting BER and NER activity. These cultures (but not the TP53-depleted cultures which arrested at the $\mathrm{G}_{1} / \mathrm{S}$ border) arrested in $\mathrm{S}$ phase at $24 \mathrm{~h}$ and had high levels of apoptosis and phospho- $\mathrm{H} 2 \mathrm{AX}$, consistent with our previous study and other studies $(29,41,42,46-48)$. ATM was activated in response to continuous high-dose 5-FU in HCT15 cultures indicating DSB formation, but CHEK2 activation at Thr68 was not observed, consistent with the fact that this cell line carries the $\mathrm{R} 145 \mathrm{~W}$ mutation (on one allele of) the $C H E K 2$ gene (26), resulting in a destabilized CHEK2 protein that cannot be phosphorylated by ATM at Thr68 (49). CHEK1 was not activated in response to either low or high doses of 5-FU, suggesting that SSBs were not formed in this cell line. An $\mathrm{S}$ phase arrest concomitant with increased levels of phosphoH2AX and high levels of (TP53-independent) apoptosis was seen in these cultures at $48 \mathrm{~h}$, indicative of a high number of DSBs that overwhelmed DNA repair capacity.

Overall, patterns of DNA damage signaling, i.e., activation of ATM, CHEK1 and/or CHEK2 reflected the formation of SSBs and/or DSBs in response to 5-FU and indicated when DNA damage had become significant (5-FU-dose-dependent). TP53 stabilization indicated its transcriptional activation and potential association with BER (and/or NER) repair pathways. Cellular responses to 5-FU-induced DNA damage were affected by both MMR and TP53 status, but MMR deficiency especially seemed to confer less sensitivity to 5-FU. Decreased cytotoxicity in MMR-deficient cells after various drug treatments is thought to reflect a tolerance to DNA damage, implying a significant role for MMR deficiency in the cellular response to 5-FU $(19,20)$. Identification of the genotypes/phenotypes involved in MMR-deficiency in colorectal tumors would have important clinical implications for tumor drug response.

\section{Acknowledgements}

This study was supported by Foundation UNI, the Norwegian Cancer Society and Medinnova.

\section{References}

1. Longley DB, Harkin DP and Johnston PG: 5-Fluorouracil: mechanisms of action and clinical strategies. Nat Rev Cancer 3: 330-338, 2003

2. Sampath D, Rao VA and Plunkett W: Mechanisms of apoptosis induction by nucleoside analogs. Oncogene 22: 9063-9074, 2003.

3. Ingraham HA, Dickey L and Goulian M: DNA fragmentation and cytotoxicity from increased cellular deoxyuridylate. Biochemistry 25: 3225-3230, 1986.

4. Mauro DJ, De Riel JK, Tallarida RJ and Sirover MA: Mechanisms of excision of 5-fluorouracil by uracil DNA glycosylase in normal human cells. Mol Pharmacol 43: 854-857, 1993.

5. Bartek J and Lukas J: DNA damage checkpoints: from initiation to recovery or adaptation. Curr Opin Cell Biol 19: 238-245, 2007.

6. Sancar A, Lindsey-Boltz LA, Unsal-Kacmaz K and Linn S: Molecular mechanisms of mammalian DNA repair and the DNA damage checkpoints. Annu Rev Biochem 73: 39-85, 2004.

7. Bartek J and Lukas J: Chk1 and Chk2 kinases in checkpoint control and cancer. Cancer Cell 3: 421-429, 2003.
8. Shiloh Y: ATM and related protein kinases: safeguarding genome integrity. Nat Rev Cancer 3: 155-168, 2003.

9. Zhou BB and Elledge SJ: The DNA damage response: putting checkpoints in perspective. Nature 408: 433-439, 2000.

10. Shechter D, Costanzo V and Gautier J: Regulation of DNA replication by ATR: signaling in response to DNA intermediates. DNA Repair (Amst) 3: 901-908, 2004.

11. Vogelstein B, Lane D and Levine AJ: Surfing the p53 network. Nature 408: 307-310, 2000.

12. Motoyama $\mathrm{N}$ and Naka K: DNA damage tumor suppressor genes and genomic instability. Curr Opin Genet Dev 14: 11-16, 2004.

13. Shieh SY, Ikeda M, Taya Y and Prives C: DNA damage-induced phosphorylation of p53 alleviates inhibition by MDM2. Cell 91: 325-334, 1997.

14. Sakaguchi K, Saito S, Higashimoto Y, Roy S, Anderson CW and Appella E: Damage-mediated phosphorylation of human p53 threonine 18 through a cascade mediated by a casein 1-like kinase. Effect on Mdm2 binding. J Biol Chem 275: 9278-9283, 2000.

15. Banin S, Moyal L, Shieh S, et al: Enhanced phosphorylation of p53 by ATM in response to DNA damage. Science 281: 1674-1677, 1998.

16. Canman CE, Lim DS, Cimprich KA, et al: Activation of the ATM kinase by ionizing radiation and phosphorylation of p53. Science 281: 1677-1679, 1998.

17. Appella E and Anderson CW: Post-translational modifications and activation of p53 by genotoxic stresses. Eur J Biochem 268: 2764-2772, 2001.

18. Tibbetts RS, Brumbaugh KM, Williams JM, et al: A role for ATR in the DNA damage-induced phosphorylation of p53. Genes Dev 13: 152-157, 1999.

19. Meyers M, Hwang A, Wagner MW and Boothman DA: Role of DNA mismatch repair in apoptotic responses to therapeutic agents. Environ Mol Mutagen 44: 249-264, 2004.

20. Meyers M, Wagner MW, Hwang HS, Kinsella TJ and Boothman DA: Role of the hMLH1 DNA mismatch repair protein in fluoropyrimidine-mediated cell death and cell cycle responses. Cancer Res 61: 5193-5201, 2001.

21. Boyer JC, Umar A, Risinger JI, et al: Microsatellite instability, mismatch repair deficiency, and genetic defects in human cancer cell lines. Cancer Res 55: 6063-6070, 1995.

22. Carethers JM, Chauhan DP, Fink D, et al: Mismatch repair proficiency and in vitro response to 5-fluorouracil. Gastroenterology 117: 123-131, 1999.

23. Ikeda M, Orimo $\mathrm{H}$, Moriyama $\mathrm{H}$, et al: Close correlation between mutations of E2F4 and hMSH3 genes in colorectal cancers with microsatellite instability. Cancer Res 58: 594-598, 1998.

24. Niewolik D, Vojtesek B and Kovarik J: p53 derived from human tumour cell lines and containing distinct point mutations can be activated to bind its consensus target sequence. Oncogene 10 : 881-890, 1995.

25. Vasilevskaya IA, Rakitina TV and O'Dwyer PJ: Geldanamycin and its 17-allylamino-17-demethoxy analogue antagonize the action of Cisplatin in human colon adenocarcinoma cells: differential caspase activation as a basis for interaction. Cancer Res 63: 3241-3246, 2003.

26. Bell DW, Varley JM, Szydlo TE, et al: Heterozygous germ line hCHK 2 mutations in Li-Fraumeni syndrome. Science 286 : 2528-2531, 1999.

27. Falck J, Lukas C, Protopopova M, Lukas J, Selivanova G and Bartek J: Functional impact of concomitant versus alternative defects in the Chk2-p53 tumour suppressor pathway. Oncogene 20: $5503-5510,2001$

28. Liu L, Nakatsuru Y and Gerson SL: Base excision repair as a therapeutic target in colon cancer. Clin Cancer Res 8: 2985-2991, 2002.

29. Adamsen BL, Kravik KL, Clausen OP and De Angelis PM: Apoptosis, cell cycle progression and gene expression in TP53depleted HCT116 colon cancer cells in response to short-term 5-fluorouracil treatment. Int J Oncol 31: 1491-1500, 2007.

30. De Angelis PM, Kravik KL, Tunheim SH, Haug T and Reichelt WH: Comparison of gene expression in HCT116 treatment derivatives generated by two different 5 -fluorouracil exposure protocols. Mol Cancer 3: 11, 2004.

31. Pizzorno G, Sun Z and Handschumacher RE: Aberrant cell cycle inhibition pattern in human colon carcinoma cell lines after exposure to 5-fluorouracil. Biochem Pharmacol 49: 553-557, 1995.

32. Vindelov LL, Christensen IJ and Nissen NI: A detergent-trypsin method for the preparation of nuclei for flow cytometric DNA analysis. Cytometry 3: 323-327, 1983. 
33. Boland CR, Koi M, Chang DK and Carethers JM: The biochemical basis of microsatellite instability and abnormal immunohistochemistry and clinical behavior in Lynch syndrome: from bench to bedside. Fam Cancer 7: 41-52, 2008.

34. Adimoolam S and Ford JM: p53 and regulation of DNA damage recognition during nucleotide excision repair. DNA Repair (Amst) 2: $947-954,2003$.

35. Seo YR and Jung HJ: The potential roles of p53 tumor suppressor in nucleotide excision repair (NER) and base excision repair (BER). Exp Mol Med 36: 505-509, 2004.

36. Zhou J, Ahn J, Wilson SH and Prives C: A role for p53 in base excision repair. EMBO J 20: 914-923, 2001.

37. Waning DL, Lehman JA, Batuello CN and Mayo LD: Controlling the Mdm2-Mdmx-p53 Circuit. Pharmaceuticals (Basel) 3 . 1576-1593, 2010.

38. Jayaraman L, Murthy KG, Zhu C, Curran T, Xanthoudakis S and Prives C: Identification of redox/repair protein Ref-1 as a potent activator of p53. Genes Dev 11: 558-570, 1997.

39. Gaiddon C, Moorthy NC and Prives C: Ref-1 regulates the transactivation and pro-apoptotic functions of p53 in vivo. EMBO J 18: 5609-5621, 1999.

40. Bulavin DV, Saito S, Hollander MC, et al: Phosphorylation of human p53 by p38 kinase coordinates N-terminal phosphorylation and apoptosis in response to UV radiation. EMBO J 18: 6845-6854, 1999.

41. Kulkarni A and Das KC: Differential roles of ATR and ATM in p53, Chk1, and histone H2AX phosphorylation in response to hyperoxia: ATR-dependent ATM activation. Am J Physiol Lung Cell Mol Physiol 294: L998-L1006, 2008.
42. Shi Z, Azuma A, Sampath D, Li YX, Huang P and Plunkett W: S-Phase arrest by nucleoside analogues and abrogation of survival without cell cycle progression by 7-hydroxystaurosporine. Cancer Res 61: 1065-1072, 2001.

43. Robinson HM, Jones R, Walker M, et al: Chk1-dependent slowing of S-phase progression protects DT40 B-lymphoma cells against killing by the nucleoside analogue 5-fluorouracil. Oncogene 25: 5359-5369, 2006.

44. Rogakou EP, Pilch DR, Orr AH, Ivanova VS and Bonner WM: DNA double-stranded breaks induce histone H2AX phosphorylation on serine 139. J Biol Chem 273: 5858-5868, 1998.

45. Sedelnikova OA, Rogakou EP, Panyutin IG and Bonner WM: Quantitative detection of (125)IdU-induced DNA double-strand breaks with gamma-H2AX antibody. Radiat Res 158: 486-492, 2002.

46. Ewald B, Sampath D and Plunkett W: Nucleoside analogs: molecular mechanisms signaling cell death. Oncogene 27: 6522-6537, 2008.

47. Furuta T, Takemura H, Liao ZY, et al: Phosphorylation of histone $\mathrm{H} 2 \mathrm{AX}$ and activation of Mre11, Rad50, and Nbs1 in response to replication-dependent DNA double-strand breaks induced by mammalian DNA topoisomerase I cleavage complexes. J Biol Chem 278: 20303-20312, 2003.

48. Sampath D, Shi Z and Plunkett W: Inhibition of cyclin-dependent kinase 2 by the Chk1-Cdc25A pathway during the S-phase checkpoint activated by fludarabine: dysregulation by 7-hydroxystaurosporine. Mol Pharmacol 62: 680-688, 2002.

49. Wu X, Webster SR and Chen J: Characterization of tumorassociated Chk2 mutations. J Biol Chem 276: 2971-2974, 2001. 